\title{
Rythme alimentaire et vidange stomacale chez le brochet (Esox lucius L.) au cours des changements de régime de la première année de croissance
}

\author{
A. Filleul ${ }^{1}$ \\ H. Le Louarn ${ }^{1}$
}

Mots clés : brochet, Esox lucius, alevin, vidange gastrique, rythme alimentaire.

La vidange stomacale et les rythmes de prise alimentaire ont été étudiés chez des brochets d'âge 0+ en suivant l'évolution de leur régime alimentaire (planctonophage, entomophage, ichtyophage). La vidange stomacale a été suivie en présentant les proies majoritairement consommées dans le milieu naturel aux stades alimentaires concernés. En général les équations linéaires et logarithmiques décrivent le mieux la vidange gastrique. Les effets sur la vidange de la température, de la nature des proies et de leur taille sont discutés. Les brochets digèrent mal la chitine : l'évolution de la digestion des proies chitineuses est présentée. A tous les stades alimentaires, les brochets montrent une activité prédatrice diurne. Le rythme alimentaire est de plus nettement biphasique au stade planctonophage.

Feeding rhythm and gastric emptying in the pike (Esox lucius L.) during the feeding changes below one year of age

Keywords : pike, Esox lucius, fingerling, gastric emptying, feeding rhythm.

The gastric emptying and the feeding rhythm have been studied with young pike below one year of age according to the natural progressive changes in their feeding process (planktivorous, entomophagous, ichthyophagous). The gastric emptying has been followed along with prey that are to be encountered by pike in their natural environment. In general, linear and logarithmic equations best describe the gastric emptying. The effects of the temperature and of the size and the nature of the prey upon the gastric emptying are dicussed. Pike do not digest chitin well. The evolution of the digestion of chitinous prey is given. At every feeding stage, pike display a day-time hunting activity. Moreover, the feeding rhythm is clearly bi-phasic at the planktivorous stage.

\section{Introduction}

En raison de son importance écologique et économique, le brochet (Esox lucius L.) se trouve régulièrement au centre de travaux scientifiques. Ces recherches ont souvent pour but de déterminer les caractéristiques quantitatives et qualitatives de son alimentation, d'une part pour estimer son activité prédatrice dans le milieu naturel, d'autre part pour maîtriser son élevage. L'étude des poissons carnassiers, dans les premiers mois de leur existence, se révèle particulièrement intéressante en raison de la succession de plu-

1. Laboratoire d'Ecologie aquatique, 65 , rue de Saint-Brieuc, F-35042 Rennes Cedex, France. sieurs phases alimentaires au cours de la croissance, chacune de ces phases étant définie par une catégorie de proie préférentielle. Le brochet présente ainsi trois stades alimentaires (planctonophage, entomophage, ichtyophage) dont les modalités temporelles sont très bien connues (Balvay 1983). De même, la nature des proies consommées n'est plus une interrogation grâce aux nombreuses analyses de contenus stomacaux déjà effectuées (Frost 1954, Treasurer \& Owen 1991, inter alia). Par contre, les variations de la vidange gastrique et du rythme alimentaire lors des différents stades n'ont pas été étudiées. Pourtant, en partant de ces données pour d'autres espèces de poissoṇs, de nombreux auteurs ont pu estimer la ration alimentaire du taxon considéré par l'utilisation de modèles mathématiques (e.g. Elliott \& Persson 1978). Nous proposons donc ici une première approche globale de ces variations en 
présentant les caractéristiques de la vidange stomacale pour les trois régimes ainsi que les rythmes alimentaires des brochetons aux stades planctonophage et entomophage. Nous proposons par ailleurs un suivi de l'état des proies à différents temps après ingestion. Cette partie purement descriptive, destinée à ceux qui étudient les contenus stomacaux, permet de retrouver l'heure d'ingestion à partir de l'état des proies.

\section{Matériel et méthodes}

Chaque stade alimentaire a nécessité l'utilisation de dispositifs expérimentaux différents.

\subsection{Stade planctonophage}

\section{Vidange gastrique}

Les expériences ont été menées dans une pièce climatisée à température constante $\left(15^{\circ} \mathrm{C}\right)$. Les alevins de brochets mesuraient de 29 à $35 \mathrm{~mm}$. Des cladocères de l'espèce Daphnia obtusa, facile à capturer selon Würtz (1945), leur étaient présentés. Les daphnies étaient préalablement calibrées par tamisage. Les brochets capturaient leurs proies immédiatement après leur déversement dans l'aquarium. Après l'ingestion de 15 daphnies, les brochets étaient isolés. En effet, au delà de la quinzième daphnie, le temps d'ingestion devenait trop long pour être compatible avec l'expérience. Les contenus stomacaux étaient prélevés par dissection à des pas de temps d'une heure (cinq brochetons par point). L'état des daphnies était noté et l'ensemble du contenu stomacal mis à l'étuve à $60^{\circ} \mathrm{C}$. L'évolution du poids sec du contenu stomacal a été suivi. Le poids initial était calculé à partir d'un échantillon de 100 daphnies de l'aquarium expérimental (poids sec des 15 daphnies: 0,001455g).

\section{Rythme alimentaire}

Une centaine de brochets âgés de 28 jours et mesurant entre 26 et $38 \mathrm{~mm}$ étaient placés dans une cage flottante de $50 \mathrm{~cm}$ de côté $\left(1 / 8^{\text {ème }}\right.$ de $\left.\mathrm{m}^{3}\right)$. Pour éviter un brusque pic de prise alimentaire lors de déversement des proies en cours d'expérience, l'ensemble du zooplancton a été fourni la veille de l'étude. Il s'agissait d'un mélange de daphnies et de copépodes du genre Cyclops, la source de daphnies pures n'étant plus disponible. L'expérience s'est déroulée sur 24 heures. A partir de 5 heures 30 du matin, puis toutes les trois heures, cinq brochets étaient capturés et tués dans un anesthésiant surdosé. Leur contenu stomacal était immédiatement extrait, observé à la loupe binoculaire, puis placé 24 heures dans une étuve à $60^{\circ} \mathrm{C}$.

En général, le niveau de réplétion est exprimé par le $\mathrm{Cr}$ (coefficient de réplétion) qui représente le rapport entre le poids frais du contenu stomacal et le poids frais du poisson exprimé en pourcentage (Grès 1995, Lejolivet 1988). Il nous est apparu préférable d'employer un indice utilisant le poids sec du contenu stomacal pour ne pas être dépendants des pertes en eau par évaporation lors des manipulations. L'indice choisi correspond au poids sec du contenu stomacal en $1 / 100$ ème de gramme sur le poids frais du poisson en $1 / 10$ ème de gramme.

\subsection{Stade entomophage}

Vidange gastrique

Les brochetons âgés de deux mois mesuraient de 6,3 à $8,5 \mathrm{~cm}$. Cinq larves de Chironomidés de taille moyenne leur était distribuées quotidiennement. Après quelques jours d'accoutumance, les brochets s'en emparaient immédiatement après la distribution. Quand le temps total d'ingestion ne dépassait pas quinze minutes, le brochet était utilisé pour l'expérience. L'heure de départ était la moyenne entre l'ingestion de la première larve et de la dernière larve. Trois brochets étaient tués pour les heures déterminées $(3 \mathrm{~h}, 6 \mathrm{~h}, 9 \mathrm{~h}$, $12 \mathrm{~h}, 15 \mathrm{~h}$ ).

L'expérience a été répétée en changeant la nature de la proie. Nous nous sommes servis du comportement cannibale du brochet et avons fourni comme proies des alevins de brochets provenant d'une ponte tardive. Pour pouvoir comparer les résultats des deux expériences, le poids des alevins de brochets distribués était du même ordre que le poids des 5 larves de Chironomidés (autour de $0,13 \mathrm{~g}$ ). Nous n'avons pu obtenir que deux points de comparaison car les alevins sont morts brutalement d'une pathologie non identifiée. Pour les deux types de proies les contenus stomacaux étaient mis à l'étuve à $60^{\circ} \mathrm{C}$.

\section{Rythme alimentaire}

Il a été étudié dans des bassins enherbés de $40 \mathrm{~m}^{2}$ subdivisés en quatre parties. Mis en eau début mars, leur équilibre physico-chimique et biologique (proies disponibles) a été suivi en continu. Ces bassins ont été empoissonnés en brochetons au stade de la résorption de la vésicule vitelline à raison de 20 individus pour $10 \mathrm{~m}^{2}$. Les poissons prélevés étaient anesthésiés puis formolés avant dissection. Le pas de temps choisi était de 3 heures et 10 brochetons par points étaient prélevés. La température était mesurée en continu. Deux expériences ont été faites au cours de la même semaine. Les contenus stomacaux étaient maintenus dans le formol dilué, avant détermination et comptage, puis pesés après passage à l'étuve. 


\subsection{Stade ichtyophage}

Les brochets, stockés individuellement dans des aquariums de 100 litres, étaient âgés de 10 mois. La difficulté essentielle était de ne pas faire subir de stress aux poissons dont l'acclimatation en aquarium devenait beaucoup plus problématique que pour les stades antérieurs. En effet, pour ne pas perturber la vidange gastrique, la prise de nourriture devait être spontanée (Windell 1978) et le gavage était à proscrire (Swenson \& Smith 1973, observations personnelles). Un abri dans l'aquarium était donc fourni aux poissons et une bâche isolait les brochets de l'observateur. L'étude s'est faite sur des brochets nourris avec des tanchettes. La vidange de brochets à qui l'on imposait un retour à un régime entomophage a également été suivie. Vingt larves de Chironomidés étaient alors fournies aux poissons, ce qui équivalait au poids d'une tanchette (environ $0,5 \mathrm{~g}$ ). L'influence de la température a aussi été testée en suivant la vidange des larves de Chironomidés à $19^{\circ} \mathrm{C}$, température à laquelle le métabolisme du brochet est optimal (Casselman 1996), et à une température de 10 degrés inférieure soit $9^{\circ} \mathrm{C}$. Trois brochets étaient utilisés par point (un point toutes les 4 heures à $19^{\circ} \mathrm{C}$, toutes les 6 heures à $9^{\circ} \mathrm{C}$ ).

\section{Résultats}

\subsection{Stade planctonophage \\ Vidange gastrique}

Pour toutes les expériences concernant la vidange gastrique, ont été testés plusieurs types de régressions (linéaire, exponentielle, puissance, logarithmique) mais seules les deux plus fortement corrélées sont présentées. Les régressions sont calculées sur l'ensemble des individus mais nous n'avons représenté que les moyennes pour chaque temps de prélèvement. L'évolution du poids sec du contenu stomacal au stade planctonophage est présentée sur la figure 1. Les deux corrélations les meilleures sont obtenues avec les régressions linéaire et logarithmique. Au bout de la cinquième heure, l'ensemble des proies a pratiquement été évacué, il ne reste qu'un résidu chitineux. Le suivi de l'état des ingesta au cours du temps est donné dans le tableau 1.

\section{Rythme alimentaire}

La courbe de l'indice de réplétion est très nettement biphasique (Fig. 2). Les deux maxima sont observés pour les prélèvements de $8 \mathrm{~h} 30$ et $20 \mathrm{~h} 30$. Ils sont liés

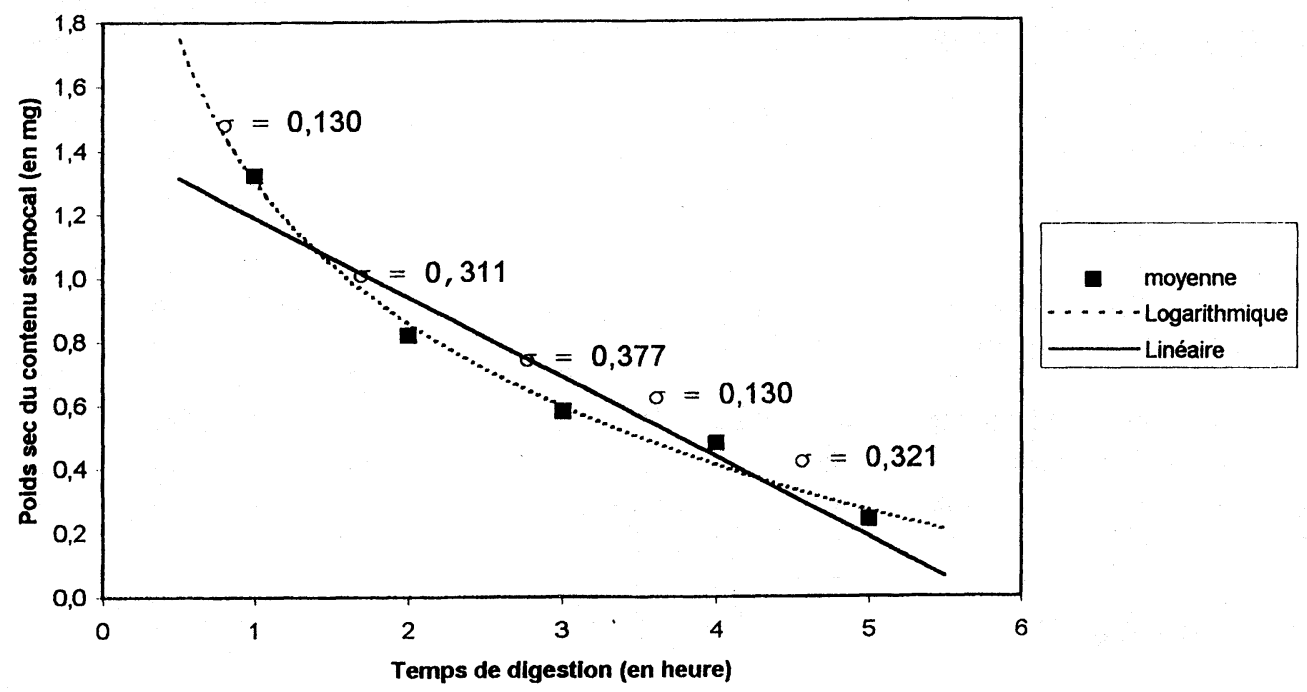

\begin{tabular}{lcc}
\hline \multicolumn{1}{c}{ Régression } & $\mathrm{R}^{2}$ & $\mathrm{ddl}$ \\
\hline Linéaire: $\mathrm{Y}=-0.25 \mathrm{X}+1.438$ & 0.6422 & 24 \\
Logarithmique: $\mathrm{Y}=-0.6416 \ln (\mathrm{X})+1.3024$ & 0.6833 & 24 \\
\hline
\end{tabular}

Fig.1. Stade planctonophage. Evolution du poids sec du contenu stomacal.

Fig. 1. Planktivorous stage. Dry weight evolution of the stomach contents. 
Tableau 1. Evolution du nombre et de l'état des daphnies.

Table 1. Number and digestion stages of Daphnia.

\begin{tabular}{|c|c|c|}
\hline $\begin{array}{l}\text { Temps après } \\
\text { l'ingestion }\end{array}$ & $\begin{array}{c}\text { Nombre moyen de } \\
\text { daphnies dans l'estomac }\end{array}$ & $\begin{array}{c}\text { Niveau } \\
\text { de digestion }\end{array}$ \\
\hline 1 heure & 13,8 & $\begin{array}{c}\text { La carapace est intacte hormis l'épine caudale, absente chez la } \\
\text { majorité des individus. Les daphnies sont, au moins } \\
\text { partiellement, vidées de leurs viscères. }\end{array}$ \\
\hline 2 heures & 9,2 & $\begin{array}{c}\text { La carapace est beaucoup plus atteinte. Elle prend un aspect } \\
\text { flasque et n'est plus intacte. Seuls la capsule céphalique, les } \\
\text { yeux, les œufs et les antennes sont à peu près intacts. On } \\
\text { observe un gradient de digestion. Les daphnies situées vers } \\
\text { l'œsophage sont beaucoup moins digérées que celles placées } \\
\text { près du sphincter gastro-intestinal. }\end{array}$ \\
\hline 3 heures & 4 & $\begin{array}{l}\text { Les daphnies sont très digérées. Il ne reste que les capsules } \\
\text { céphaliques et des fragments de carapace. Les yeux, les œufs } \\
\text { et quelques antennes sont encore visibles. }\end{array}$ \\
\hline 4 heures & 4 & Mêmes caractéristiques que le point précédent. \\
\hline 5 heures & 0 & Il ne reste que des œufs ou des capsules céphaliques isolés. \\
\hline
\end{tabular}

au lever et au coucher du soleil ( 6 h 45 et 21 h15). Le premier marque la reprise d'alimentation après une nuit d'inactivité, suivie d'une phase de digestion et d'activité prédatrice moindre. Après cette phase qui dure de 3 à 6 heures, les brochets reprennent leur activité prédatrice qui culmine au crépuscule. La nuit entraîne l'arrêt de la consommation. L'état des proies atteste de leur ingestion plus ou moins antérieure à

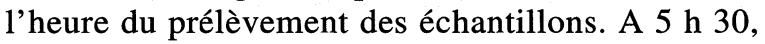
l'ensemble du contenu stomacal est digéré et il est difficile de reconnaître la nature de la proie. Ceci apparaît comme la confirmation qu'aucune ingestion n'avait eu lieu récemment et qu'il n'y a pas d'activité prédatrice nocturne. Trois heures plus tard, on trouve dans les estomacs des proies peu digérées qui témoignent d'une prise récente de nourriture. Le suivi de la vidange stomacale indique que des proies de cet état ont fait un séjour d'au plus trois heures dans les estomacs. L'évolution au cours du temps du nombre moyen de proies fraîchement ingérées (Fig. 2) est représentée par une courbe à nouveau biphasique, d'aspect très semblable à la première, ce qui nous permet d'être sûr que l'augmentation du poids dans les estomacs, correspondant à une certaine tranche horaire dépend de l'ingestion récente de proies et non de nombreux résidus d'organismes ingérés plus antérieurement. Enfin, le fait que le poids des contenus stomacaux prélevés à $5 \mathrm{~h} 30$ soit relativement important, et ce malgré l'absence de proies fraîches dans les estomacs suggère que la vidange stomacale se fait moins vite durant la nuit.

\subsection{Stade entomophage}

\section{Vidange stomacale (Fig. 3)}

Quand les proies fournies sont des larves de Chironomidés, les régressions logarithmique et exponentielle sont les plus fortement corrélées aux points obtenus. Après une phase de digestion rapide, un plateau se dessine à la 9 ème heure. Au bout de 15 heures, $85 \%$ de la quantité a été vidangée. Ce point marque la fin de l'expérience, une période de faible activité digestive se dessinant. Il est en effet admis qu'en l'absence d'un nouveau repas persiste dans les estomac une faible quantité d'ingesta (Windell 1978). Nous avons suivi l'état des ingesta (Fig. 4A) en attribuant 3 niveaux de digestion pour chaque larve de Chironomidés :

- niveau $1:$ la larve est intacte ;

- niveau $2:$ la larve est vidée mais le tégument est intact ;

- niveau 3 : le tégument est attaqué.

Après trois heures de digestion, il reste encore des larves intactes. De plus, ce temps apparaît insuffisant pour attaquer l'enveloppe cuticulaire. On retrouve donc l'intégralité des 15 larves dans l'ensemble des contenus stomacaux. Comme pour les daphnies au stade précédent, on observe une première phase de digestion des organes internes suivie de la digestion plus difficile du tégument. Après 6 heures, la fragmentation du tégument a commencé. Il ne reste que quelques enveloppes fragmentées après 9 heures. Enfin, après 15 heures, le contenu stomacal n'est plus constitué que par un résidu sans organe reconnaissable. 


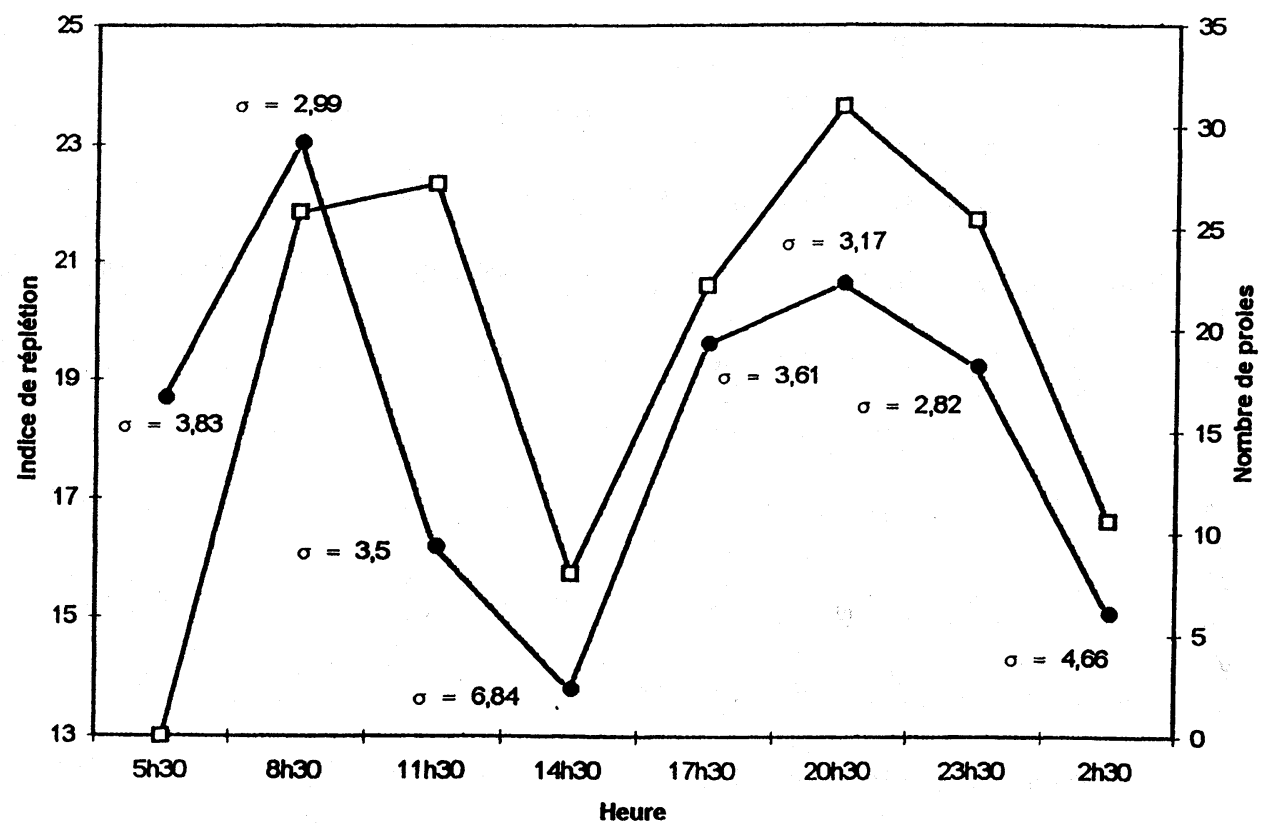

-Indice de réplétion - - -Nombre de proies nouvellement ingérées

Fig.2. Rythme alimentaire au stade planctonophage. Température $13^{\circ} \mathrm{C}$ à $19^{\circ} \mathrm{C}$.

Fig. 2. Feeding rhythm at the planktivorous stage. Temperature $13^{\circ} \mathrm{C}$ to $19^{\circ} \mathrm{C}$.

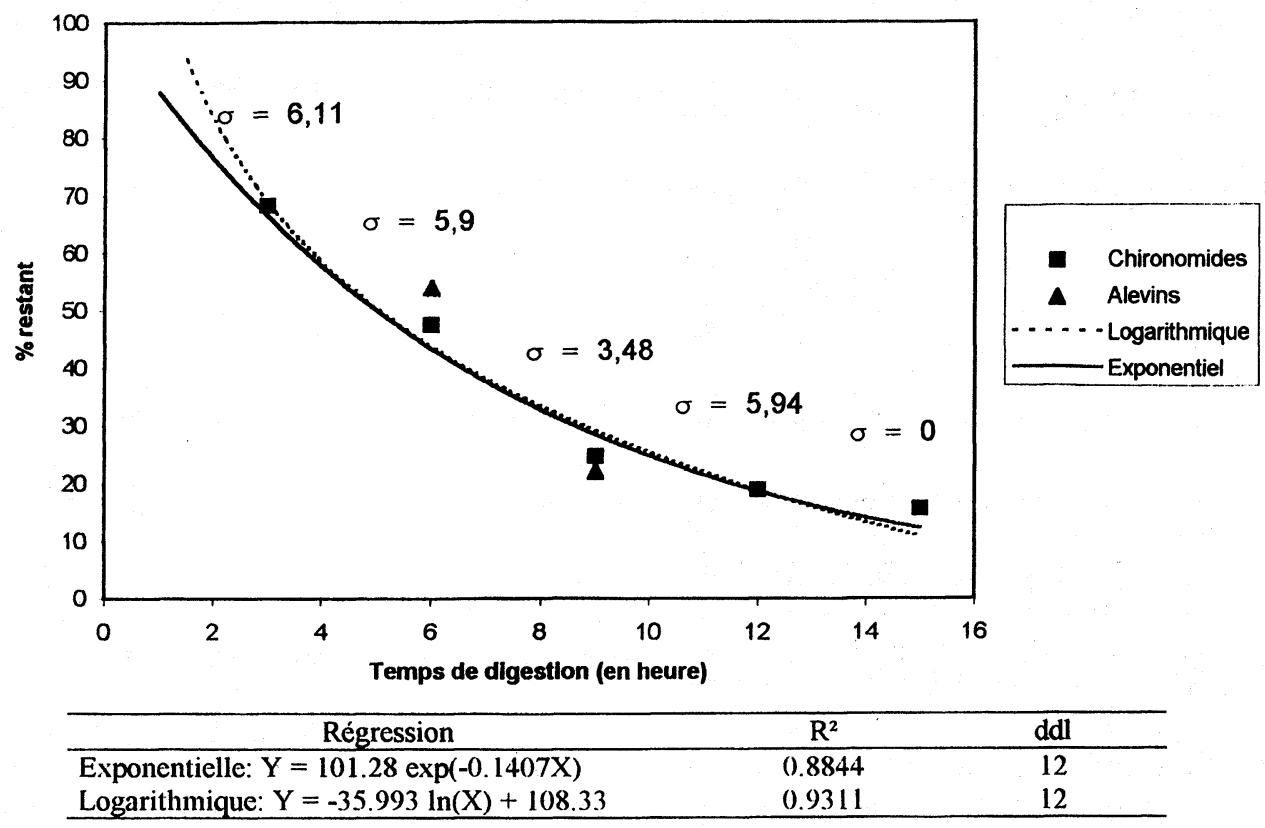

Fig.3. Stade entomophage. Evolution du poids sec du contenu stomacal.

Fig. 3. Entomophagous stage. Stomach content dry weight evolution. 
Quand les proies fournies sont des alevins de brochet, les quantités vidangées sont comparables à celles obtenues avec les larves de Chironomidés. Les deux points obtenus sont illustrés avec la courbe précédente (Fig. 3).

\section{Rythme alimentaire}

Pour le calcul de l'indice de réplétion deux séries de prélèvements ont eu lieu à 8 jours d'intervalle. Les proies consommées par les brochets, parmi celles naturellement disponibles dans la mare, sont les suivantes (en pourcentage d'occurence): larves de Chironomidés $(88 \%)$, Cladocères $(67 \%)$, Ostracodes $(49 \%)$. Les résultats sont reportés sur la figure 5 . Les deux séries de prélèvements affichent certes une bimodalité mais moins nette qu'au stade planctonophage. Les écartstypes sont de plus très forts : contrairement au stade antérieur, la prise de nourriture n'est pas synchrone. L'observation de l'état de digestion des proies nous permet de dire qu'il n'y a pas non plus d'activité prédatrice nocturne au stade entomophage. En effet, la nuit (prélèvements à $24 \mathrm{~h}$ et $3 \mathrm{~h}$ ) l'estomac est occupé par un culot de digestion. Celui ci s'élimine après le lever du jour lorsque la consommation reprend. Le soir est un moment de forte activité alimentaire pour les deux séries de prélèvements.

\subsection{Stade ichtyophage}

Pour ce test ont été choisis des brochetons de $15 \mathrm{~cm}$, taille atteinte en fin de premier été. A cette taille, les brochets peuvent être, suivant le milieu concerné, ichtyophages ou encore en grande partie entomophage (Munro 1957, observations personnelles). Seule la vidange stomacale a été étudiée en fournissant aux brochets des alevins de tanches à $9^{\circ} \mathrm{C}$ (Fig. 6), des larves de Chironomides à $19^{\circ} \mathrm{C}$ (Fig. 7) et $9^{\circ} \mathrm{C}$ (Fig. 8). Pour les trois séries de données les régressions linéaire et logarithmique sont les plus fortement corrélées. Mais l'évacuation de la tanche s'adapte mieux à une régression linéaire alors que les larves de Chironomidés s'évacuent selon une fonction logarithmique pour les deux températures considérées. A $9^{\circ} \mathrm{C}$, la quantité vidangée après trente heures est à peu près identique pour les deux types de proies. Ceci confirme que la vidange stomacale est moins influencée par la nature de la proie que par son volume (le même dans les deux expériences). L'augmentation de la température accélère la vidange stomacale: le pourcentage d'ingesta restant dans les estomacs est plus faible au bout de 16 heures à $19^{\circ} \mathrm{C}$ qu'après 30 heures de digestion à $9^{\circ} \mathrm{C}$. On s'aperçoit aussi en suivant l'évolution de la dégradation des cuticules (Fig 4B et 4C) que la digestion n'est pas seulement plus rapide mais aussi plus complète.
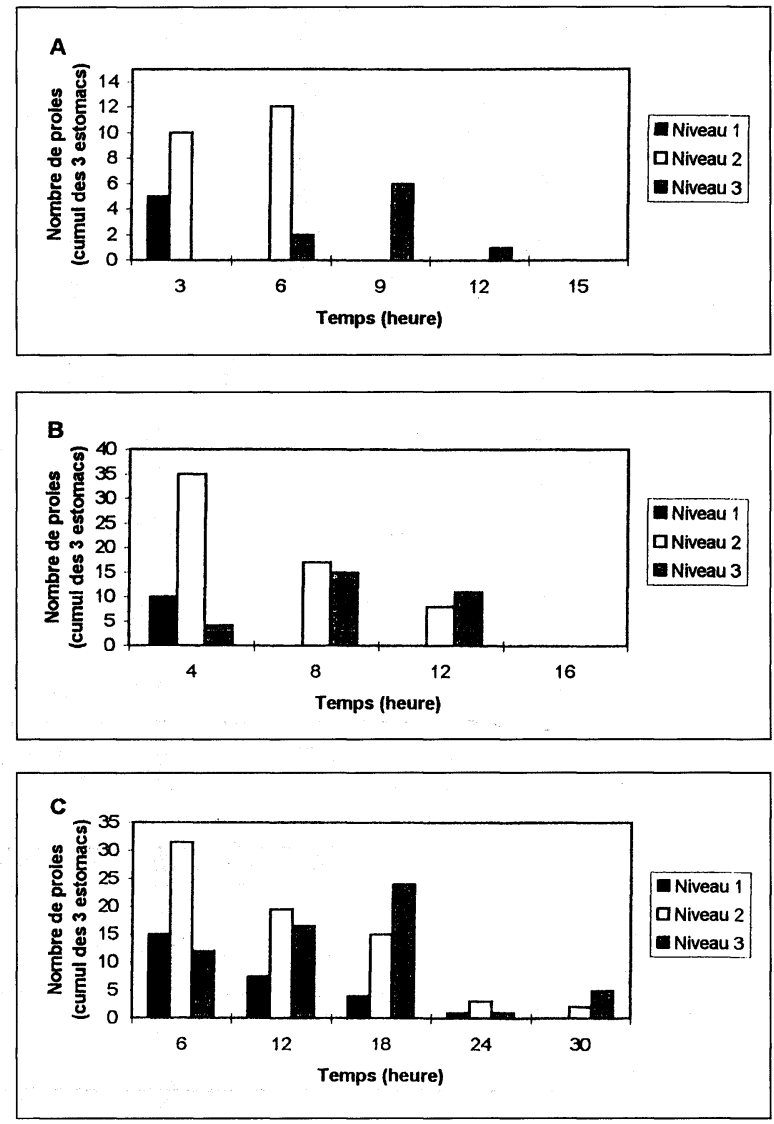

Fig.4. Evolution du nombre et de l'état des larves de Chironomidés. A- Stade planctonophage, à $15^{\circ} \mathrm{C}$ B- Stade ichtyophage, à $19^{\circ} \mathrm{C}$ C- Stade ichtyophage, à $9^{\circ} \mathrm{C}$

Fig. 4. Evolution of the number and condition of Chironomids larvae.
A- Planktivorous stage, at $15^{\circ} \mathrm{C}$
B- Ichtyophagous stage, at $19^{\circ} \mathrm{C}$
C- Ichtyophagous stage, at $9^{\circ} \mathrm{C}$

Après 12 heures de digestion à $19^{\circ} \mathrm{C}$, il ne reste plus aucune enveloppe cuticulaire même morcelée.

\section{Discussion et conclusion}

\section{Vidange gastrique}

Les caractéristiques de la vidange gastrique des poissons dépendent des proies consommées. Jobling $(1981,1986)$ a prouvé que le niveau énergétique, le degré de digestibilité et la taille de la proie affectaient la vidange qui pouvait être linéaire ou curviligne. Le plus souvent, pour nos tests, le poids sec des contenus stomacaux décroit avec le temps selon une fonction linéaire ou logarithmique. La régression linéaire était la plus corrélée quand les brochets consommaient des 


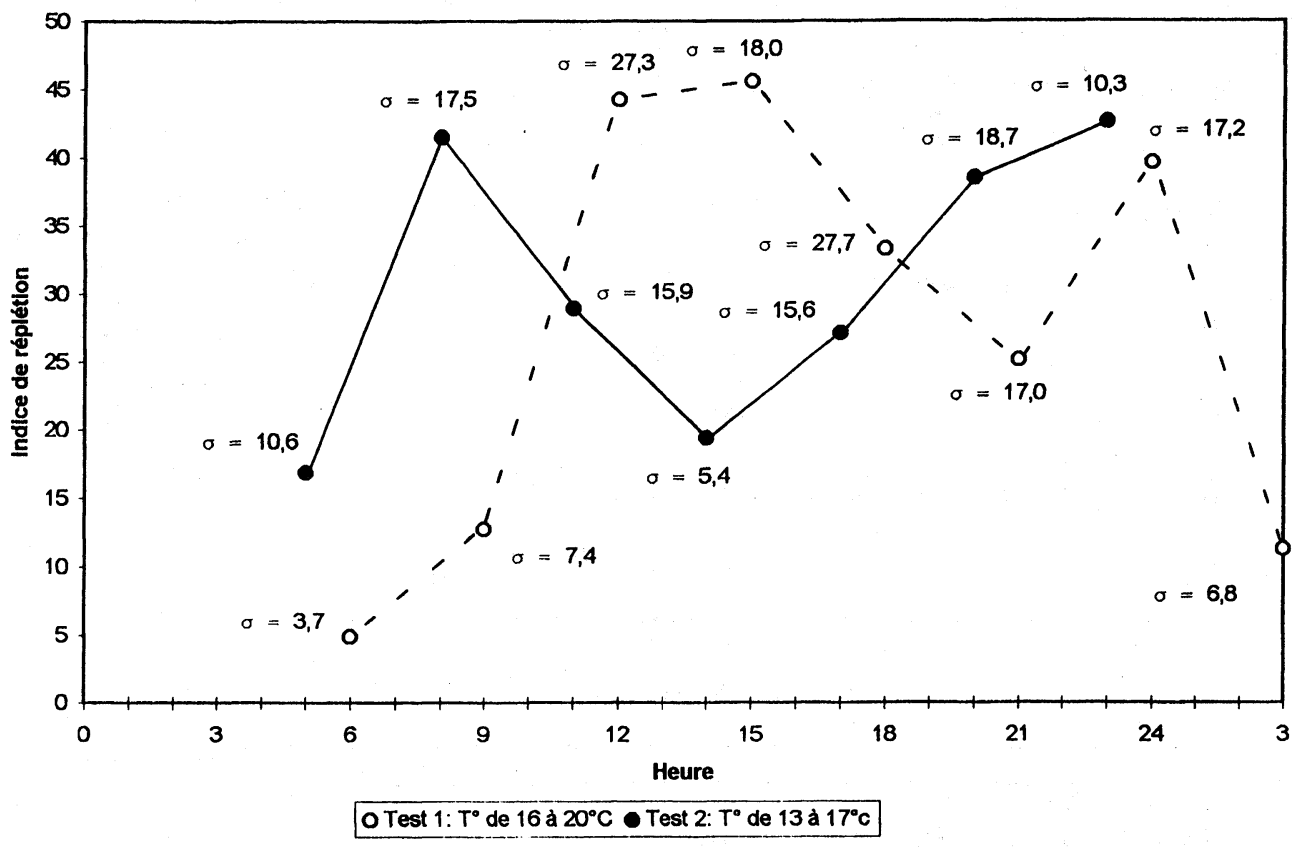

Fig.5. Rythme alimentaire au stade entomophage.

Fig. 5. Feeding rhythm at the entomophageous stage.

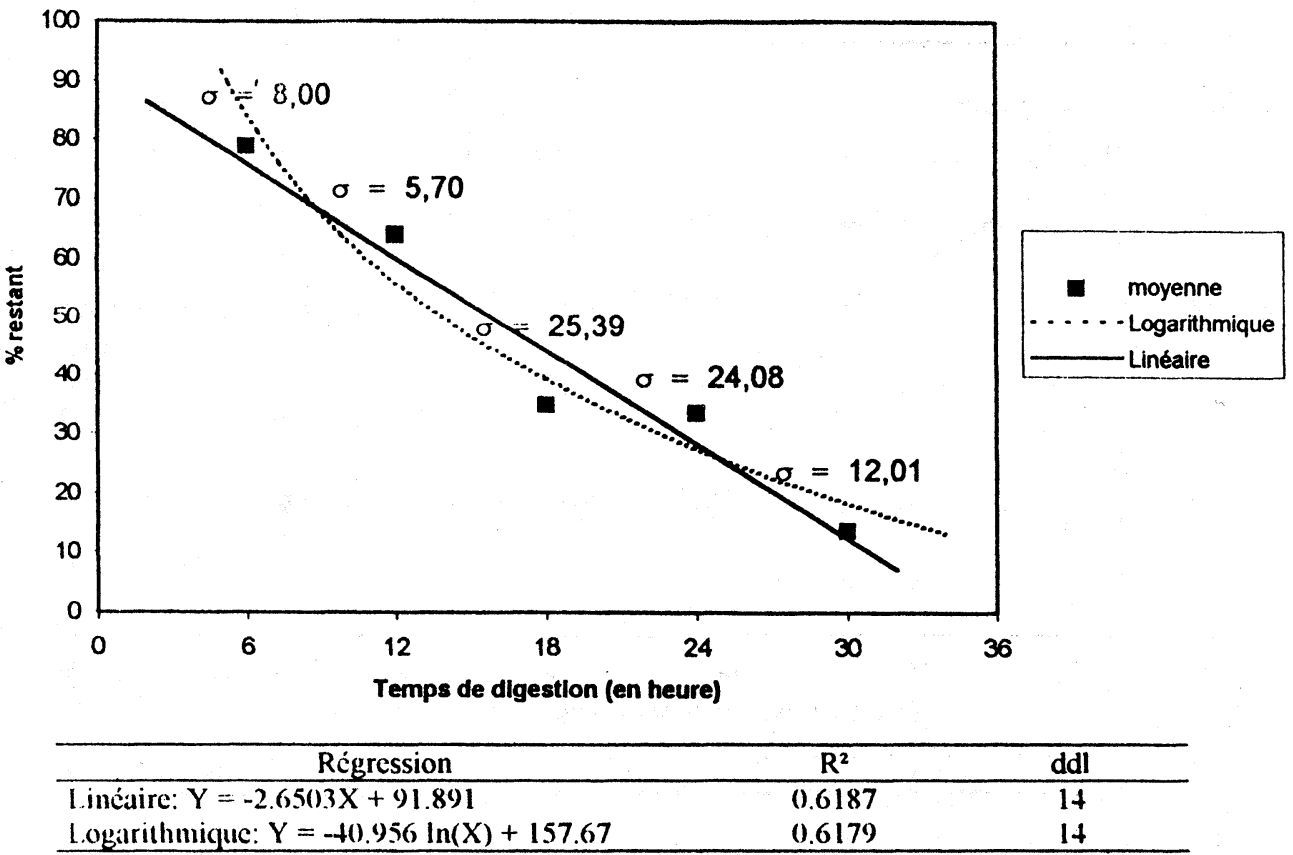

Fig.6. Vidange stomacale des brochets de $15 \mathrm{~cm}$ nourris de tanches à $9^{\circ} \mathrm{C}$.

Fig. 6. Gastric emptying of $15 \mathrm{~cm}$ long pike fed with tenches at $9^{\circ} \mathrm{C}$. 


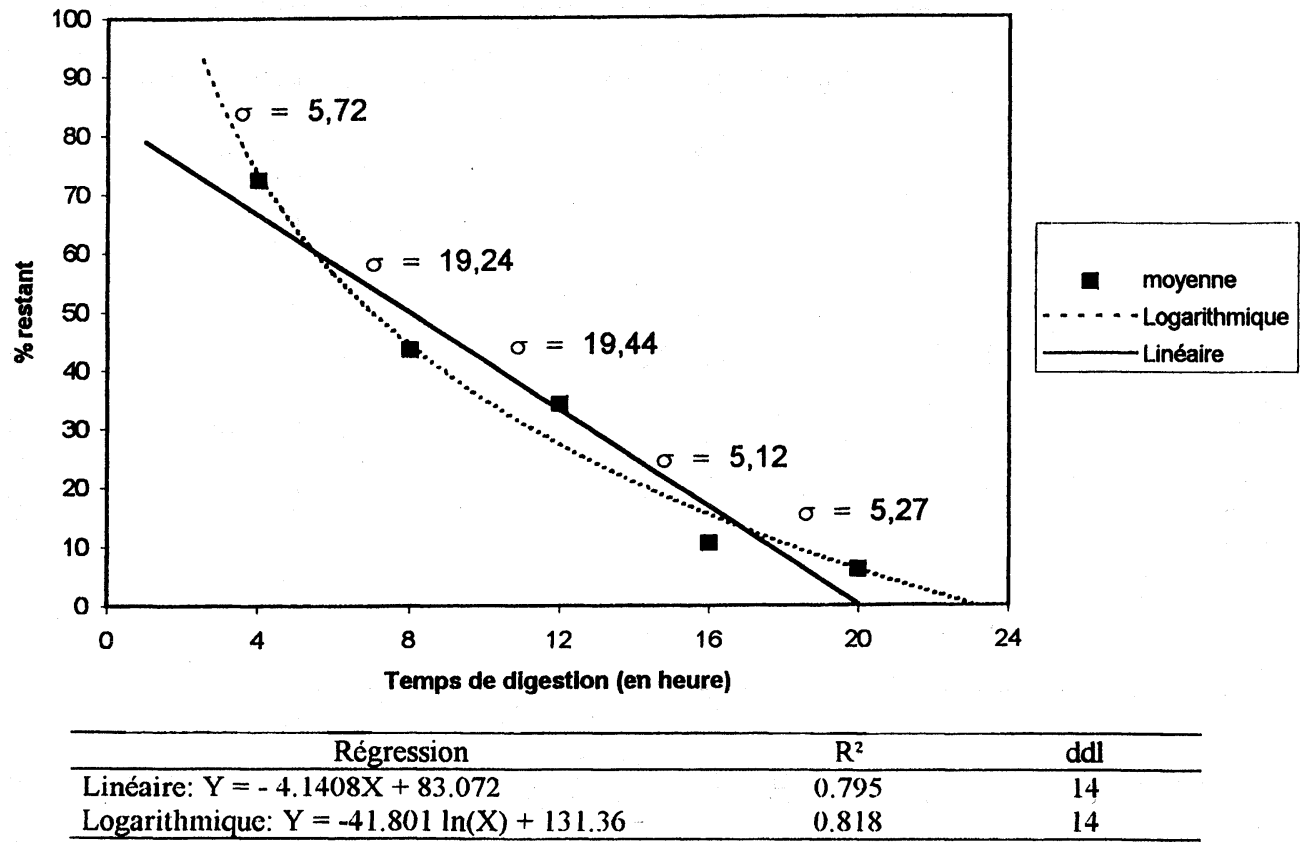

Fig.7. Vidange stomacale des brochets de $15 \mathrm{~cm}$ nourris de larves de Chironomidés à $19^{\circ} \mathrm{C}$.

Fig. 7. Gastric emptying of $15 \mathrm{~cm}$ long pike fed with Chironomid larvae at $19^{\circ} \mathrm{C}$.

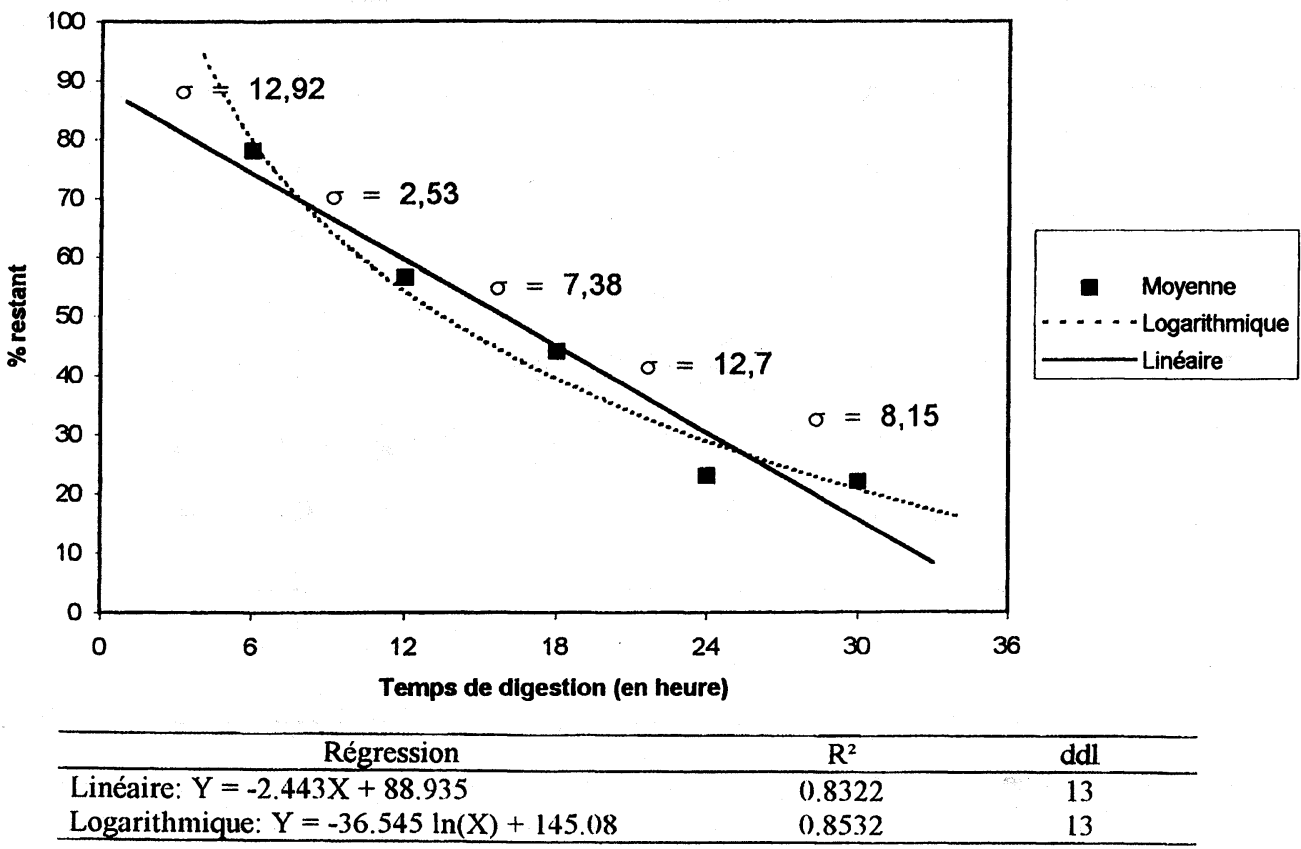

Fig. 8. Vidange stomacale des brochets de $15 \mathrm{~cm}$ nourris de larves de Chironomidés à $9^{\circ} \mathrm{C}$.

Fig. 8. Gastric emptying of $15 \mathrm{~cm}$ long pike fed with Chironomid larvae at $9^{\circ} \mathrm{C}$. 
tanches: comme l'ont signalé Jobling (1986) et Smith et al. (1989), la vidange des grosses proies se fait plutôt selon une fonction linéaire et celle des petites proies de façon curviligne. Précisons que pour d'autres auteurs, c'est la fonction puissance (Talbot et al. 1984) ou exponentielle (Elliott 1991) qui est la mieux adaptée.

Il est apparu que les quantités vidangées par unité de temps étaient proportionnellement moindre au fur et à mesure que le brochet grandissait et cela quel que soit le type d'aliment. Ainsi, chez les brochets de deux mois, nourris avec des larves de Chironomidés, la vidange est beaucoup plus longue qu'au stade précédent alors que le rapport Poids des ingesta / Poids des brochets était plus faible $(6 \%$ du poids vif contre $11 \%$ pour les brochets planctonophages). Cette particularité ne provient vraisemblablement pas de la nature différente des proies. En effet, Luquet \& Luquet (1983), observaient des différences comparables pour deux lots de brochets nourris avec le même aliment (nourriture composée). D'après ces mêmes auteurs, ce phénomène s'accompagne d'une prise journalière de nourriture proportionnellement plus faible. Cette évolution du métabolisme et du comportement est directement liée à la croissance de l'animal qui monopolise l'essentiel de la nourriture ingérée. Les besoins d'entretien du brochet sont en effet très faibles (Johnson 1966). Les besoins relatifs des alevins diminuent donc avec l'augmentation de la taille.

La digestibilité différente des proies n' a pas fait varier le temps de vidange (identique pour les Chironomidés et les tanchettes à $9^{\circ} \mathrm{C}$ et pour les Chironomidés et les alevins de brochets à $\left.15^{\circ} \mathrm{C}\right)$. Il est d'ailleurs couramment admis que c'est la distention de l'estomac qui modifie le taux d'évacuation gastrique et de nombreux auteurs ont proposé des modèles présentant le taux d'évacuation comme une fonction du volume d'ingesta (voir la liste des modèles dans Stubb 1977).

La température accélère très nettement la vidange stomacale. Cette observation est conforme aux résultats de Salam \& Davies (1994) qui montraient une augmentation de la consommation avec l'augmentation de la température quelles que soient les classes de taille considérées. Chez les poissons, tandis que la croissance est caractérisée par une température optimale, la vidange tend à s'accélérer avec l'augmentation de température pour atteindre une valeur maximale à l'approche de la limite de tolérance de l'espèce (Brett \& Higgs 1970, Elliott 1972). Trois processus au moins sont dépendants de la température: l'activité enzymatique, la sécrétion des sucs gastriques, la motricité stomacale (Smit 1966).
Les proies ne se digèrent pas de façon homogène: les brochets digèrent plus difficilement les cuticules de crustacés et de larves d'insectes qui sont composées de $68 \%$ à $85 \%$ de chitine (Colin \& Pérès 1971). L'hydrolyse de la chitine demande un système enzymatique spécifique qui nécessite deux enzymes: une chitinase et une chitobiase (Devigné \& Jeuniaux 1961, in Colin $\&$ Pérès 1971). Le brochet serait dépourvu de ce système enzymatique (Pérès 1979) ce qui expliquerait que l'on peut trouver des proies imparfaitement digérées à la sortie du tube digestif. Il existe en général une adaptation du tube digestif et de ses sécrètions au régime alimentaire (Kapoor et al. 1975) mais tous les poissons ne peuvent pas digérer la chitine. Rösch (1985) a montré que la chitine des daphnies n'était pas digérée par les juvéniles de Coregonus lavaretus (L.).

Les différences d'état de digestion sont souvent utilisées comme indicateurs des pics alimentaires (Hyslop 1980). Le suivi de la dégradation des proies que nous proposons pour les trois stades considérés devrait s'avérer utile à ceux qui étudient le rythme alimentaire.

\section{Le rythme alimentaire}

La courbe obtenue pour le stade planctonophage correspond tout à fait aux observations de Lejolivet (1988) et Grès (1994) qui précisent que dans le milieu naturel, on observe souvent deux pics de réplétion, un diurne et un crépusculaire. Néanmoins, des dissimilitudes apparaissent: Lejolivet remarquait que des alevins élevés avec une très forte ration n'avaient pas de rythme de prise alimentaire marqué ce qui n'a pas été le cas dans notre cage où le plancton était pourtant en très grande densité. De plus, le pic diurne observé par Lejolivet, nettement plus tardif, se situait entre 15 et 18 heures et le dernier pic pouvait être nocturne $(3 \mathrm{~h})$. Ce dernier résultat va à l'encontre des résultats de Grès (1995) qui remarquait la cessation immédiate de l'action de chasse une fois que les brochets étaient plongés dans l'obscurité. Les décalages entre les pics peuvent s'expliquer par des différences de condition d'éclairement qui peuvent créer un «effet silhouette» des proies devenant plus visibles par contraste (Hall et al. 1979). De plus, le rythme de prédation dépend aussi du rythme d'activité des proies (Dobler 1977).

Au stade entomophage, en se référant à la fois à l'indice de réplétion et à l'état de digestion, la prédation est à nouveau synchronisée avec la phase éclairée du nycthémère. Le rythme alimentaire apparaît cependant beaucoup plus irrégulier et asynchrone.

$\mathrm{Au}$ terme de cette étude, nous pouvons dire que pour les scénarios testés, la vidange gastrique du brochet suit les caractéristiques observées chez d'autres pois- 
sons dans des travaux antérieurs. Le rythme alimentaire biphasique évoque, quant à lui, les résultats obtenus avec d'autres poissons carnassiers comme la perche Perca fluviatilis L. et la perchaude Perca flavescens (Keast \& Welsh 1968, Fortin \& Magnin 1972). Cette étude présente des résultats exploitables à la fois pour les modélisateurs et les écologistes de terrain.

\section{Travaux cités}

Balvay G. 1983. - L'alimentation naturelle des alevins de brochet (Esox lucius L.) durant leur premier mois de vie. In : Le brochet : gestion dans le milieu naturel et élevage, R. Billard (Ed.), INRA Publ : 179-198.

Brett J. R. \& Higgs D. A. 1970. - Effect of temperature on the rate of gastric digestion in fingerling Sockeye Salmon, Oncorhynchus nerka. J. Fish. Res. Board Can., 27 : 1767-1779.

Casselman J. M. 1996. - Age, growth and environmental requirements of pike. In : Pike, biology and exploitation, John F. Craig Ed. : 70-100.

Colin D. A. \& Peres G. 1971. — Etude comparée des systèmes enzymatiques chitinolytiques du tube digestif de quelques téléostéens marins. Ann. Inst. Michel Pacha, 4 : 1-9.

Dobler E. 1977. - Correlation between the feeding time of the pike (Esox lucius) and the dispersion of a school of Leucaspices delineatus. Oecologia, 27 : 93-96.

Elliott J.M. 1972. - Rate of gastric evacuation in brown trout, Salmo trutta, L. Freshwat. Biol., 2 : 1-18.

Elliott J M. \& Persson L. 1978. - The estimation of daily rates of food consumption for fish. J. Anim Ecol., 47 : 977-991.

Elliott J.M. 1991. - Rates of gastric evacuation in piscivorous brown trout, Salmo trutta. Freshwat. Biol., 25 : 297-305.

Fortin R. \& Magnin E. 1972 - Quelques aspects qualitatifs et quantitatifs de la nourriture des perchaudes, Perca flavescens, dans la grande Anse de l'île de Perrot, au lac St-Louis. Ann. Hydrobiol., 3 :79-91.

Frost W.E. \& Kipling C. 1967. — A study of reproduction, early life, weight length relationship and growth of pike, Esox lucius L., in Windermere. J. Anim. Ecol., $36: 651-693$.

Grès P. 1994. - Production intensive de brochetons (Esox lucius L.) nourris de proies vivantes issues de bassins de lagunage naturel. Survie, croissance, alimentation. Thèse de Doctorat, Université Blaise Pascal Clermont-Ferrand : 247 p. + annexes.

Grès P. 1995. - Aspects quantitatifs de l'alimentation zooplanctonophage de brochets (Esox lucius L., 1758) en élevage intensif. Icthyophysiologica Acta, $18: 1-21$.

Hall D.J., Werner E.E., Gilliam J.F., Mittelbach G.G., Howard D., Doner C.G., Dickerman J.A. \& Stewart A.J. 1979. - Diel foraging behavior and prey selection in the golden shiner (Notemigonus crysoleucas). J. Fish. Res. Board Can., 36 : 1029-1039.

Hyslop E.J. 1980. - Stomach contents analysis-a review of methods and their application. J. Fish Biol.,17 : 411-429.
Jobling M. 1986. - Mathematical models of gastric emptying and implications for food consumption studies. Env. Biol. Biol. Fish., $16: 35-50$.

Jobling M. 1981. - Dietary digestibility and the influence of food components on gastric evacuation in plaice, Pleuronectes platessa L.. J.Fish Biol., 19 : 29-36.

Johnson L. 1966. - Experimental determination of food comsumption of pike, Esox lucius L., for growth and maintenance. J. Fish. Res. Board., Can., 23 : 1495-1505.

Kapoor B.G., Smith H. \& Verighina I.A. 1975. - The alimentary canal and digestion in teleosts. Adv. Mar. Biol., $13: 109-239$.

Keast A. \& Welsh L. 1968 - daily feeding periodicities, food uptake rates, and dietary changes with hour of day in some lake fishes. J. Fish. Res. Board Can., 25 : 1133-1144.

Lejolivet C. 1988. - Croissance et comportement alimentaire d'alevins de brochets (Esox lucius L.) élevés en cages dans le réservoir de Pareloup. Annls Limnol., 24 : 183-192.

Luquet P. \& Luquet J.F. 1983 - Appréciation du niveau d'ingestion et de la vitesse du transit alimentaire chez l'alevin de brochet nourri avec de l'aliment composé. In : Gestion dans le milieu naturel et élevage, R. Billard Ed. Le Brochet, INRA Publ. : 235-243.

Munro W.R. 1957. - The pike of Loch Choin. Freshw. and Salmon Fish. Research., $16: 2-16$;

Perès G. 1979. - Les protéases, l'amylase, les enzymes chitinolytiques, les laminarases. In : Nutrition des poissons, M. Fontaine (Ed.), Acte du colloque CNERNA, Edition du CNRS : 55-64.

Rösch R. 1987. - Chitin as a marker used for the determination of the utilization of Daphnia pilicaria fed to Coregonus lavaretus. Proc. V Congr. Europ. Ichthyol., Stockholm 1985, 339-343.

Salam A. \& Davies P.M.C. 1994. - Effect of body weight and temperature on the maximum daily food consumption of Esox lucius. J. Fish. Biol., 44 : 167-169.

Smit H. 1966.- Influence of temperature on the rate of gastric juice secretion in the brown bullhead. Ictalarus nebulosus. Comp. Biochem. Physiol., 21 : 125-132.

Smith R.L., Paul J.M. \& Paul A.J. 1989. - Gastric evacuation in Walleye pollock, Theragra chalcogramma. Can. J. Fish. Aquat. Sci., 46 : 489-493.

Stubbs D.F. 1977. - Models of gastric emptying, Gut, 18 : 202-207.

Swenson W.A. \& Smith L.L. 1973. - gastric digestion, food consumption, feeding periodicity and food conversion efficiency in walleye (Stizostedion vitreum vitreum). Board Can., 30 : 13271336.

Talbot C., Higgings P.J. \& Shanks A.M. 1984. — Effects of pre- and postprandial starvation on meal size and evacuation rate of juvenile Atlantic Salmon, Salmo salar L. J. Fish. Biol., 25 : 551-560.

Treasurer J. \& Owen R. 1991. — Food and growth of pike, Esox lucius in simple fish communities in lakes of different trophic status. Aquat. Living Resour., 4 : 289-292.

Windell T. 1978. - Digestion and the daily ration of fishes, 159183. In : Ecology of freshwater fish production, Gerking (Ed.), Oxford : $520 \mathrm{p}$.

Würtz A. 1945. - Developpement, biologie et nutrition des jeunes alevins de brochet (Esox lucius L.). Bull. Fr. Piscic., 135 : 57-69. 